\author{
Kensuke Ochi · Yataro Daigo · Toyomasa Katagiri \\ Akihiko Saito-Hisaminato · Tatsuhiko Tsunoda \\ Yoshiaki Toyama $\cdot$ Hideo Matsumoto \\ Yusuke Nakamura
}

\title{
Expression profiles of two types of human knee-joint cartilage
}

Received: 16 December 2002 / Accepted: 27 December 2002/Published online: 21 February 2003

(C) The Japan Society of Human Genetics and Springer-Verlag 2003

\begin{abstract}
We have performed a comprehensive analysis of gene-expression profiles in human articular cartilage (hyaline cartilage) and meniscus (fibrocartilage) by means of a cDNA microarray consisting of 23,040 human genes. Comparing the profiles of the two types of cartilage with those of 29 other normal human tissues identified 24 genes that were specifically expressed in both cartilaginous tissues; these genes might be involved in maintaining phenotypes common to cartilage. We also compared the cartilage profiles with gene expression in human mesenchymal stem cells (hMSC), and detected 22 genes that were differentially expressed in cells representing the two cartilaginous lineages, 11 specific to each type, which could serve as markers for predicting the direction of chondrocyte differentiation. Our data should also provide useful information about regeneration of cartilage, especially in support of efforts to identify cartilage-specific molecules as potential agents for therapeutic approaches to joint repair.
\end{abstract}

K. Ochi · Y. Daigo · A. Saito-Hisaminato · Y. Nakamura $(\square)$ Laboratory of Molecular Medicine,

Human Genome Center, Institute of Medical Science, University of Tokyo, 4-6-1 Shirokanedai,

Minato-ku, Tokyo 108-8639, Japan

E-mail: yusuke@ims.u-tokyo.ac.jp

Tel.: + 81-3-5449-5372

Fax: +81-3-5449-5433

T. Katagiri · Y. Nakamura

Laboratory of Genome Technology,

Human Genome Center,

Institute of Medical Science,

University of Tokyo, Tokyo, Japan

T. Tsunoda

Laboratory for Medical Informatics, SNP Research Center, Institute of Physical and Chemical Research (Riken),

Kanagawa, Japan

K. Ochi · Y. Toyama $\cdot$ H. Matsumoto

Department of Orthopaedic Surgery,

Keio University School of Medicine,

Tokyo, Japan
Keywords Hyaline cartilage - Fibrocartilage ·

Expression profiles $\cdot$ Marker gene $\cdot$ Regenerative medicine

\section{Introduction}

Several transcriptional factors are thought to be key factors directing differentiation of mesenchymal tissues: molecules of the MYOD family for muscle differentiation (Ferrari et al. 1998), PPARG2 for adipose tissue differentiation (Rosen and Spiegelman 2000), and CBFA1 for osteogenic differentiation (Komori et al. 1997). Although SOX9 and CBFA1 are thought to be key transcriptional factors during cartilage differentiation (Bi et al. 1999), other factors are likely to be involved as well (Takeda et al. 2001). Articular cartilage (hyaline cartilage) of the knee, and the knee meniscus (fibrocartilage), are different (Ross et al. 1995). Identification of common cis-elements in genes expressed specifically in these two types of cartilage should yield insight into cartilage-specific transcriptional regulation.

Investigators interested in cartilage development and regeneration have a few markers available for predicting the direction of differentiation, i.e., type II collagen and aggrecan for hyaline cartilage, and type I collagen for fibrocartilage (Burgeson et al. 1982; Ross et al. 1995; Carlberg et al. 2001). Cartilaginous cells are believed to originate from multipotential mesenchymal cells that resemble human mesenchymal stem cells (hMSCs) (DeLise et al. 2000; Carlberg et al. 2001). Since hMSCs are relatively easy to obtain, hMSC cultures are considered to be good sources of cells for modeling regeneration of mesenchymal tissues such as cartilage and bone (Pittenger et al. 1999; Carlberg et al. 2001; Ochi et al. 2003). Thus, genes that are expressed specifically in either hyaline cartilage or fibrocartilage should be useful markers to indicate the direction of differentiation. To identify such genes, we have applied a cDNA microarray 
containing 23,040 genes in a system that provides highthroughput analysis of expression. In the work reported here, we evaluated the power of this system to detect cartilage-specific molecules.

\section{Materials and methods}

\section{Clinical samples}

All patients were diagnosed and treated by members of the Department of Orthopedic Surgery, Keio University School of Medicine. "Normal" articular cartilage samples were obtained during total knee replacement surgery performed on eight female patients (age range 56-80 years, median 69 years) who were diagnosed with primary osteoarthritis of the knee. In each case a nonaffected site of articular cartilage, such as the patellar-femoral joint, was carefully peeled to provide samples for further investigations. "Normal" meniscus tissues were obtained from samples removed during endoscopic meniscectomy of six patients (five male and one female; age range 13-28 years, median 20 years) who were diagnosed with traumatic meniscal injury; again, non-affected tissue corresponding to each sample was obtained for our investigations. All 14 patients (or their families in some cases) gave informed consent according to the guidelines of both Keio University and the University of Tokyo. Resected samples were snap-frozen in liquid nitrogen immediately and sent to the Human Genome Center, Institute of Medical Science at the University of Tokyo, without any personal identifiers.

\section{Preparation, T7-based amplification, and labeling of RNAs}

Total RNA was extracted from each frozen clinical sample and from human mesenchymal stem cells (hMSC) purchased from BioWhittaker, Inc. (Walkersville, MD) to serve as a universal control, using TRIzol reagent (Life Technologies, Inc., Rockville, MD). Total RNAs of 29 normal human tissues (mesenteric adipose tissue, bone marrow, brain, heart, kidney, liver, lung, lymph node, mammary gland, pancreas, placenta, prostate, salivary gland, skeletal muscle, small intestine, spinal cord, spleen, stomach, testis, thymus, thyroid, trachea, uterus, colon, ovary, fetal brain, fetal kidney, fetal liver, and fetal lung) were obtained as described previously (Saito-Hisaminato et al. 2002). Equal amounts of RNA from each of the 29 normal tissues were pooled to serve as another universal control (29Mix). All RNA preparations were digested with RNase-free DNase I (Nippon Gene Co., Tokyo, Japan) according to the manufacturer's recommendations. Two rounds of T7-based RNA amplification and the preparation of cDNA probes were carried out as described elsewhere (Kitahara et al. 2001; Ochi et al. 2002). Amplified RNA $(2.5 \mu \mathrm{g})$ from each cartilage sample (articular cartilage: a mixture of 8 cases; meniscus, 6 patients) was labeled with Cy5-dCTP (Amersham Biosciences, Uppsala, Sweden); an equal amount of amplified RNA from each universal control (29Mix or a pool of amplified RNA from hMSCs) was labeled with Cy3-dCTP (Amersham Biosciences) for hybridization to the cDNA microarray.

\section{Construction and analysis of the cDNA microarray}

Fabrication of our cDNA microarray slides has been described previously (Ono et al. 2000; Kitahara et al. 2001). Slides containing a duplicate set of 23,040 cDNA spots were used for each analysis of expression profiles, to reduce experimental fluctuation. Hybridizations were performed to compare hyaline cartilage (Cy5) with 29Mix (Cy3), fibrocartilage (Cy5) with 29Mix (Cy3), hyaline cartilage (Cy5) with hMSC (Cy3), and fibrocartilage (Cy5) with hMSC (Cy3). Hybridization, washing, and scanning were performed as described elsewhere (Ono et al. 2000; Kitahara et al.
2001). The intensity of each duplicated signal was evaluated photometrically using the ArrayVision computer program (Imaging Research, Inc., St Catharines, Ontario, Canada). Intensities of the 29 individual normal tissues (test) and 29Mix (control) were evaluated as described elsewhere (Saito-Hisaminato et al. 2002). The fluorescence intensities of Cy5 (test) and Cy3 (control) for each target spot were adjusted so that the mean Cy5/ $\mathrm{Cy} 3$ ratio of the 52 housekeeping genes was equal to one. We assigned a cut-off value to each microarray slide, using variance analysis. If both $\mathrm{Cy} 3$ and $\mathrm{Cy} 5$ signal intensities were less than the cut-off values, the expression level of the corresponding gene in that sample was assessed as low or absent. For other genes we calculated $\mathrm{Cy} 5 / \mathrm{Cy} 3$ as a relative expression ratio. A two-dimensional hierarchical clustering method was performed as described before (Saito-Hisaminato et al. 2002).

\section{Semi-quantitative RT-PCR}

A 3- $\mu$ g aliquot of each RNA to be used for microarray analysis was reverse-transcribed to single-stranded cDNA using oligo(dT) $)_{12-18}$ primer and Superscript II (Invitrogen, Carlsbad, CA). Semiquantitative reverse transcription PCR (RT-PCR) was carried out for seven selected genes as described previously (Ono et al. 2000), using the following primer sets: for UBA52, 5'-GAT CTT TGT GAA GAC CCT CAC TG- ${ }^{\prime}$ and $5^{\prime}$-CAG ATC ATC TTG TCG CAG TTG TA-3'; for RPL23, 5'-CTG TAT ATC ATC TCC GTG AAG GG-3' and $5^{\prime}$-AAG TCT GCA CAC TCC TTT GCT AC-3'; for $R A B 13,5^{\prime}$-CAA CAA CAC TTA CAT CTC CAC CA-3' and $5^{\prime}$-GAG CAC TTG TTG GTG TTC TTC TT-3'; for $I G F 2,5^{\prime}$-CTT GGA CTT TGA ATC AAA TTG G-3' and 5'-CCT CCT TTG GTC TTA CTG GG-3'; for $I G L @, 5^{\prime}$-CAA CTG TAC ACC TAA AGG CTC TC- $3^{\prime}$ and $5^{\prime}$-ATC TGA GAG GAG AGG AGA GTG A-3'; for $P D L I M 1,55^{\prime}$-CCA TAC AAG ATG AAT TTA GCC TCT G-3' and $5^{\prime}$-GTC AGA CAC GTT ATA TT TGA TTG GG-3'; and for COL1A1, 5'-CAT GTT CGG TTG GTC AAA GATA-3' and 5'-AAT ACA AAA CCA CCA AGA CCT CC-3'. PCR reactions were optimized for the number of cycles to ensure product intensity within the linear phase of amplification.

\section{Results and discussion}

Identification of genes that were highly expressed in both hyaline cartilage and fibrocartilage

Comparison of expression profiles in cartilage with those of other normal human tissues should indicate genes that are preferentially expressed in cartilage. We selected 6707 genes for which data were present for hyaline cartilage; 585 of them, including five collagen genes, showed hyaline cartilage/29Mix ratios $>10$, and 209 also showed hyaline cartilage/hMSC ratios $>10$ (data not shown). Similarly, we selected 6436 genes for which data were present for fibrocartilage; 605 showed fibrocartilage/29Mix ratios $>10$ and 145 showed fibrocartilage/hMSC ratios $>10$ (data not shown).

\section{Identification of "cartilage-specific" genes}

Articular cartilage and meniscus are both considered to be cartilaginous tissues (Ross et al. 1995). A hierarchical clustering of the 31 normal human tissues we examined, including the two types of cartilage, indicated that the 
latter are very closely related in gene expression profiles (data not shown). Thus we assumed that some phenotypes common to hyaline cartilage and fibrocartilage should be maintained by the same "cartilage-specific transcriptional regulators."

To obtain candidates for these putative "cartilagespecific transcriptional regulators," we searched for genes whose relative expression ratio for either hyaline cartilage/29Mix or fibrocartilage/29Mix were $>5$ while (i) expression ratios in the 29 non-cartilaginous tissues were $<2$ or (ii) expression was absent in $90 \%$ of the normal tissues, and identified 69 genes. Among them, 24 revealed expression ratios of $>5$ for both hyaline cartilage/29Mix and fibrocartilage/29Mix; but 22 of the 69 were highly expressed only in hyaline cartilage and 23 were highly expressed only in fibrocartilage (Fig. 1a, b).

Identification of separate marker genes for hyaline cartilage and fibrocartilage

Comparison of expression profiles of articular cartilage (hyaline cartilage) and meniscus (fibrocartilage) with the profile obtained from a mixture of 29 other normal tissues showed a specific character for cartilaginous tissue after final differentiation, but this approach was not sufficient for identifying genes important for understanding development and regeneration of mesenchymal tissues. Thus, we re-examined the expression profiles to investigate genes that showed different levels of expression between each type of cartilage and hMSCs. We first selected 4837 genes for which data were obtained for either hyaline cartilage/hMSC or fibrocartilage/hMSC and then looked for candidate marker genes that could satisfy the criteria of both "hyaline cartilage/hMSC ratio of $>2$ " and "fibrocartilage/hMSC ratio of $<0.5$," or genes that would satisfy the criteria of both "fibrocartilage/hMSC ratio of $>2$ " and "hyaline cartilage/hMSC ratio $<0.5$." We detected 22 genes that were, by this definition, differentially expressed in one or the other of the two types of cartilaginous cells (Fig. 2a, b).

In all, we examined 92,160 gene expression patterns in two cartilaginous tissues, in comparison with 668,160 measurements of genes expressed in 29 other normal tissues, by means of a cDNA microarray. To our knowledge this is the first study to apply such a largescale approach for analyzing expression profiles of cartilaginous tissues. This work has established an expression database for sets of genes that are expressed specifically in cartilage.

We subjected the expression profiles of two types of cartilaginous tissue and 29 normal tissues to a hierarchical clustering analysis. The dendrogram showed that articular cartilage and meniscus fell into the same, closely related terminal branch, indicating that both hyaline cartilage and fibrocartilage were very similar in gene expression patterns. Among several mesodermal tissues included in the 31-tissue panel, only bone marrow and nerve tissues yielded expression patterns that were relatively similar to that of cartilage (data not shown). Our data imply that significant alterations in expression level must occur for a large number of genes during the differentiation process.

Transcriptional regulation of genes in a given tissue is controlled by a combination of mechanisms, for example changes in chromatin structure mediated by the association of histone acetylases and deacetylases (Wade 2001), binding of transcriptional regulators to an enhancer and/or a suppressor (Luscher and Larsson 1999), binding of transcription factors to a promoter region, and methylation of $\mathrm{CpG}$ islands in promoter sequences (Ballestar and Wolffe 2001). Although the precise mechanisms of cooperation among these elements are not well understood (Cha et al. 2000), identification of common cis-elements that exist only in cartilage-specific genes should yield insight into the physiology of chondrocytes. Such core sequences might be useful for (a) identifying novel transcription-related factors in cartilage, and (b) establishing cartilage-specific expression vectors for genes that are necessary for regenerating and/or maintaining phenotypes of cartilaginous tissues.

In our experiments, 24 genes were expressed specifically in both hyaline cartilage and fibrocartilage. Among them, $W N T 7 A$ is member of the $W N T$ gene family, which consists of structurally related genes encoding secreted signaling molecules. This gene is implicated in the regulation of limb chondrogenesis (Rudnicki and Brown 1997; DeLise et al. 2000; Tufan et al. 2002). COMP is normally expressed at high levels in the territorial matrix of chondrocytes and is believed to belong to the family of thrombospondin genes (Newton et al. 1994); mutations of COMP have been found in patients with pseudoachondroplasia or multiple epiphyseal dysplasia (Ikegawa et al. 1998), indicating its important role in maintaining cartilaginous structure (Thur et al. 2001). GLG1 encodes a conserved membrane sialoglycoprotein present in the Golgi apparatus of most cells; this protein binds with basic fibroblast growth factor (FGFB) and is expressed during chondrogenesis (Stieber et al. 1995). GPS2 encodes a 327-amino-acid polypeptide with no similarity to other proteins or known motifs; however, this molecule potently regulates RAS- and mitogenactivated protein kinase (MAPK)-mediated signals and interferes with JNK activity in yeast and mammalian cells (Spain et al. 1996). Considering that $W N T$ genes, $C O M P, F G F B, M A P K$, and $J N K$ are known to play important roles in chondrogenesis (Thur et al. 2001), their products might well be critical to the physiology of chondrocytes. SOX9, believed to be a key transcriptional factor in chondrocytes (Bi et al. 1999), is a member of the sex-determining region Y-type $\mathrm{HMG}$ (high mobility group) box protein family; it is required for chondrocyte-specific gene expression and chondrogenesis (Bi et al. 1999). In our experiments the SOX9 gene was highly expressed in both hyaline cartilage and fibrocartilage (relative ratios $>10$ ), but its expression was relatively ubiquitous (relative ratio $>2$ in seven of the 29 normal non-cartilaginous tissues examined). 
Fig. 1a, b Schema of "cartilagespecific genes." Relative expression ratios were (i) $>5$ in cartilaginous tissues, while ratios in a mixture of cDNAs from 29 other normal tissues were $<2$ or (ii) $>5$ in cartilaginous tissues, while ratios were missing for $90 \%$ of individual normal tissues compared with the 29Mix. a Twenty-four of the 69 genes were highly expressed in both hyaline cartilage and fibrocartilage (overlapping), while 22 were highly expressed in hyaline cartilage and 23 were highly expressed in fibrocartilage. Each cell in the matrix represents the expression level of a single transcript in a single tissue, with red and green indicating transcript levels respectively above and below the median for that gene across the 29Mix. Black represents unchanged expression; gray indicates slight expression or none (intensities of both $\mathrm{Cy} 3$ and Cy5 less than the cut-off value). Abbreviations: $H$, relative expression ratio in hyaline cartilage; $F$, relative expression ratio in fibrocartilage. b Semiquantitative RT-PCR of three selected genes using RNAs from two types of cartilage and six other normal tissues. The integrity of each template was controlled through amplification of $\beta 2 \mathrm{MG}$
Cartilage specific genes (Overlapping)

\begin{tabular}{lllll} 
H & F Accession & HSID & Symbol & Gene Name \\
\hline \hline & L32137 & 1584 & COMP & cartilage oligomeric matrix protein \\
W51788 & 5798 & PELO & pelota (Drosophila) homolog \\
X05232 & 83326 & MMP3 & matrix metalloproteinase 3 (stromelysin 1, progelatinase) \\
AJ224677 & 7122 & SCRG1 & scrapie responsive protein 1 \\
AA780308 & 98785 & KSP37 & Ksp37 protein \\
Al022360 & 190583 & ESTs & ESTs \\
D79205 & 300141 & RPL39 & ribosomal protein L39 \\
AA127483 & 180139 & SMT3H2 & SMT3 (suppressor of mif two 3, yeast) homolog 2 \\
U70735 & 15591 & MOV34 & COP9 subunit 6 (MOV34 homolog, 34 kD) \\
U53476 & 72290 & WNT7A & wingless-type MMTV integration site family, member 7A \\
AA292179 & 119502 & UBA52 & ubiquitin A-52 residue ribosomal protein fusion product 1 \\
M74905 & 79396 & MPG & N-methylpurine-DNA glycosylase \\
AF037261 & 33787 & SCAM & vinexin beta (SH3-containing adaptor molecule) \\
U28963 & 296940 & GPS2 & G protein pathway suppressor 2 \\
X16135 & 2730 & HNRPL & heterogeneous nuclear ribonucleoprotein L \\
M81758 & 46038 & SCN4A & sodium channel, voltage-gated, type IV, alpha polypeptide \\
W84565 & 109494 & SPUF & secreted protein of unknown function \\
A1018632 & 150101 & LAMP1 & lysosomal-associated membrane protein 1 \\
AA338449 & 110695 & ESTS & ESTs, Weakly similar to B0495.6 [C.elegans] \\
U28811 & 78979 & GLG1 & Golgi apparatus protein 1 \\
AA703770 & 4888 & SARS & seryl-tRNA synthetase \\
X76013 & 79322 & QARS & glutaminyl-tRNA synthetase \\
L07540 & 171075 & RFC5 & replication factor C (activator 1) 5 (36.5kD) \\
AA292834 & 46901 & KIAA1462 & KIAA1462 protein
\end{tabular}

Cartilage specific genes (Hyalin cartilage)

H F Accession HSID Symbol Gene Name

$\begin{array}{llll}\text { U97276 } & 77266 & \text { QSCN6 } & \text { quiescin Q6 }\end{array}$

$\begin{array}{llll}\mathrm{X} 55954 & 234518 & R P L 23 & \text { ribosomal protein } \mathrm{L} 23\end{array}$

AA609358 $5245 \quad$ FLJ20643 hypothetical protein FLJ20643

Al022075 12210 FLJ13732 hypothetical protein FLJ13732 similar to tensin

Al039548 187899 ESTs ESTs

AA812932 123515 ESTs Homo sapiens cDNA FLJ13840 fis, clone THYRO1000783

AA854476 284294 BCRP2 Breakpoint cluster region protein, uterine leiomyoma, 2

Al193935 131250 LOC63920 transposon-derived Buster3 transposase-like

AA836860 287776 OTRPC4 vanilloid receptor-related osmotically activated channel

AF075292 49585 FGF18 fibroblast growth factor 18

AF038603 129758 PSTPIP1 proline-serine-threonine phosphatase interacting protein 1

L02321 75652 GSTM5 glutathione S-transferase M5

BE614190 $23495 \quad$ FLJ11252 hypothetical protein FLJ11252

AA843531 100002 HSPC162 HSPC162 protein

U05877 177559 IFNGR2 interferon gamma receptor 2 (interferon gamma transducer 1)

$\begin{array}{llll}\text { X91349 } 3759 & \text { USP5 } & \text { ubiquitin specific protease } 5 \text { (isopeptidase T) }\end{array}$

Al004706 182364 ESTs ESTs, Weakly similar to $25 \mathrm{kDa}$ trypsin inhibitor [H.sapiens]

BE502540 171077 ESTs $\quad$ ESTs, Weakly similar to similar to serine/threonine kinase [C.elegans]

X54412 154850 COL9A1 collagen, type IX, alpha 1

X75450 279651 MIA melanoma inhibitory activity

J02984 133230 RPS15 ribosomal protein S15

X56932 119122 RPL13A ribosomal protein L13a

Cartilage specific genes (Fibrocartilage)

\begin{tabular}{|c|c|c|c|c|}
\hline H F & Accession & HSID & Symbol & Gene Name \\
\hline & $\mathrm{X05345}$ & 77798 & HARS & histidyl-tRNA synthetase \\
\hline & N20283 & 26276 & ESTS & ESTs \\
\hline & W02928 & 9933 & ALTE & Ac-like transposable element \\
\hline & AA232823 & 283728 & PCNP & PEST-containing nuclear protein \\
\hline & AA780074 & 122706 & ESTS & ESTs \\
\hline & Al079967 & 117266 & ESTS & ESTs \\
\hline & AA503921 & 299121 & ESTS & ESTs \\
\hline & AA521154 & 105500 & ESTS & ESTs \\
\hline & AA214125 & 78103 & NAP1L4 & nucleosome assembly protein 1 -like 4 \\
\hline & $\times 75593$ & 151536 & RAB13 & RAB13, member RAS oncogene family \\
\hline & X97544 & 20716 & TIM17 & translocase of inner mitochondrial membrane 17 (yeast) homolog $\mathrm{A}$ \\
\hline & M94054 & 102267 & LOX & lysyl oxidase \\
\hline & J00306 & 12409 & SST & somatostatin \\
\hline & BF688836 & 77886 & LMNA & lamin A/C \\
\hline & AA583019 & 31791 & ACYP2 & acylphosphatase 2 , muscle type \\
\hline & AA766527 & 306127 & ESTS & ESTs \\
\hline & N24911 & 5258 & C11ORF2 & chromosome 11 open reading frame 2 \\
\hline & AA284317 & 6120 & FLJ13222 & hypothetical protein FLJ13222 \\
\hline & L37042 & 283738 & CSNK1A1 & casein kinase 1, alpha 1 \\
\hline & L20970 & 172081 & PDE4D & phosphodiesterase 4D, cAMP-specific \\
\hline & AA813363 & 193784 & ESTS & Homo sapiens mRNA; cDNA DKFZp586K1922 \\
\hline & AF043472 & 47584 & KCNS3 & potassium voltage-gated channel, delayed-rectifier, subfamily S, member 3 \\
\hline & AA234909 & 181077 & KIAA1306 & KIAA1306 protein \\
\hline
\end{tabular}




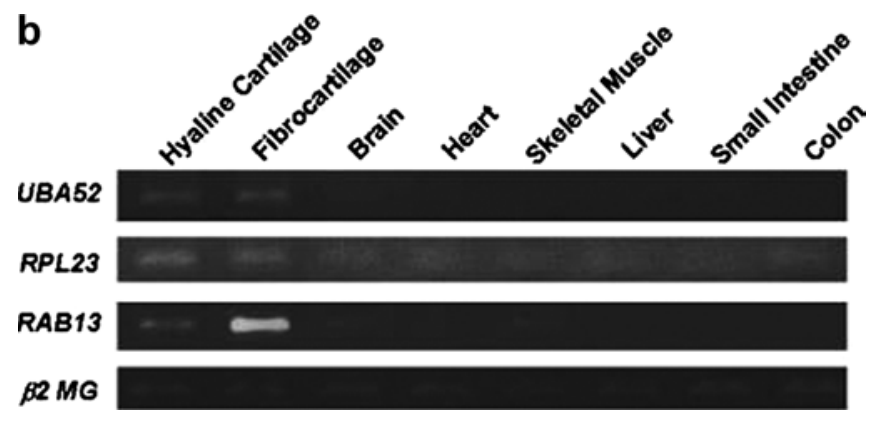

Fig. 1a, b (Continued)

Several known markers are commonly invoked to predict the direction of differentiation in studies of cartilage development and regeneration, specifically collagen type II and aggrecan for hyaline cartilage and type I collagen for fibrocartilage (Burgeson et al. 1982). Our genome-wide cDNA microarray analyses revealed 22 additional genes that were specifically expressed in cartilaginous tissue: 11 in hyaline cartilage and 11 others in fibrocartilage. RT-PCR experiments also supported differences in gene expression patterns among hyaline cartilage, fibrocartilage, and hMSC, indicating that this set of 22 genes can include candidates to serve as markers during chondrogenesis from multipotential mesenchymal cells.

Our data indicated high expression of type I collagen in fibrocartilage, but expression of this gene was very low in hyaline cartilage. On the other hand, type II collagen was extremely abundant in hyaline cartilage (ratio of $>100$ ), and relatively so in fibrocartilage (ratio of 15). The consistency of our data with previous reports verifies their quality (Tufan et al. 2002). One of the main goals of regenerative medicine is to replace damaged
Fig. 2a, b Schema of marker genes for hyaline cartilage or fibrocartilage. Relative expression ratios were $>2$ in either type, and the ratios in the other cartilage were $<0.5$ compared with hMSC, for all 22 genes listed. a Eleven of the 22 genes meeting the strict differential criteria were highly expressed only in hyaline cartilage $(\mathrm{H})$, while 11 others were highly expressed only in fibrocartilage $(\mathrm{F})$. The color scale was constructed from expression ratios of cartilaginous tissue (Cy5):hMSC (Cy3) as described in Fig. 1. b Semi-quantitative RT-PCR of four selected genes using RNAs from two types of cartilage and from hMSCs. The integrity of each template was controlled through amplification of $\beta 2 \mathrm{MG}$

\begin{tabular}{|c|c|c|c|c|}
\hline \multicolumn{5}{|c|}{ Marker genes of hyaline cartilage and fibrocartilage } \\
\hline H $\mathbf{F}$ & Accession No. & HSID & Symbol & Gene Name \\
\hline & X07868 & 251664 & IGF2 & insulin-like growth factor 2 (somatomedin A) \\
\hline & Al197946 & 181125 & IGL@ & immunoglobulin lambda locus \\
\hline & AA436362 & 30868 & RTN4R & reticulon 4 receptor (Nogo receptor) \\
\hline & AA779724 & 122113 & ESTs & ESTs \\
\hline & L05779 & 113 & $E P H \times 2$ & epoxide hydrolase 2 , cytoplasmic \\
\hline & AA809819 & 5710 & CREG & cellular repressor of E1A-stimulated genes \\
\hline & AA812932 & 123515 & FLJ13840 & Homo sapiens cDNA FLJ13840 fis, clone THYRO1000783 \\
\hline & AA761311 & 211563 & $B C L 7 A$ & B-cell CLL/lymphoma 7A \\
\hline & M22430 & 76422 & PLA2G2A & phospholipase A2, group IIA (platelets, synovial fluid) \\
\hline & AW167729 & 10029 & CTSC & cathepsin C \\
\hline & X00129 & 76461 & RBP4 & retinol-binding protein 4 , interstitial \\
\hline & AF070616 & 3618 & HPCAL1 & hippocalcin-like 1 \\
\hline & $\mathrm{Al} 268231$ & 130829 & ESTs & ESTs \\
\hline & Al183533 & 300823 & LIMK2 & LIM domain kinase 2 \\
\hline & AA316112 & 284194 & FLJ20831 & hypothetical protein FLJ20831 \\
\hline & $\mathrm{Al} 265770$ & 75807 & PDLIM1 & PDZ and LIM domain 1 (elfin) \\
\hline & Al349804 & 133294 & ESTs & ESTs \\
\hline & AA491502 & 97199 & C1QR & complement component $\mathrm{C} 1 \mathrm{q}$ receptor \\
\hline & AA977821 & 172928 & COL1A1 & collagen, type I, alpha 1 \\
\hline & AA780074 & 122706 & ESTs & ESTs \\
\hline & J03464 & 179573 & COL1A2 & collagen, type I, alpha 2 \\
\hline & AF037335 & 5338 & CA12 & carbonic anhydrase XII \\
\hline
\end{tabular}

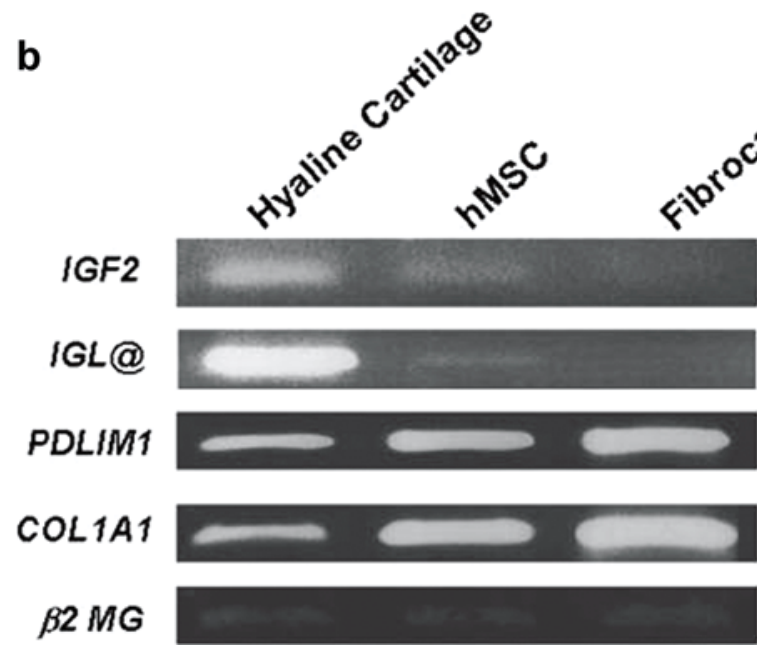


tissue with regenerated tissue that can function normally. To realize this goal it is important to establish methods to generate the required tissue as well as to maintain the physiological function of the regenerated tissue. The data documented here should provide useful information for both approaches and contribute to research toward regeneration of cartilage tissues in clinical settings.

Acknowledgements We thank Hiroko Bando, Noriko Sudo, Kie Naito, Saori Osawa, Keiko Shigeta, Mika Sanjo, Emiko Takahashi, and Miwako Ando for fabrication of the cDNA microarray; Emi Ichihashi for analysis of the data; Drs Junya Toguchida, Takashi Toyoda, and Hideki Imabayashi for helpful discussions; and Drs Toshiro Otani, Yasuo Suda, Takashi Toyoda, Masanori Imabayashi, and Taku Yatabe for preparing clinical samples. This work was supported in part by Research for the Future Program Grant No. 00L01402 from the Japan Society for the Promotion of Science.

\section{References}

Ballestar E, Wolffe AP (2001) Methyl-CpG-binding proteins: targeting specific gene repression. Eur J Biochem 268:1-6

Bi W, Deng JM, Zhang Z, Behringer RR, de Crombrugghe B (1999) Sox9 is required for cartilage formation. Nat Genet 22:85-89

Burgeson RE, Hebda PA, Morris NP, Hollister DW (1982) Human cartilage collagens: comparison of cartilage collagens with human type V collagen. J Biol Chem 257:7852-7856

Carlberg AL, Pucci B, Rallapalli R, Tuan RS, Hall DJ (2001) Efficient chondrogenic differentiation of mesenchymal cells in micromass culture by retroviral gene transfer of BMP-2. Differentiation 67:128-138

Cha JY, Kim H, Kim KS, Hur MW, Ahn Y (2000) Identification of transacting factors responsible for the tissue-specific expression of human glucose transporter type 2 isoform gene: cooperative role of hepatocyte nuclear factors 1alpha and 3beta. J Biol Chem 275:18358-18365

DeLise AM, Fischer L, Tuan RS (2000) Cellular interactions and signaling in cartilage development. Osteoarthr Cartilage 8:309-334

Ferrari G, Cusella-De Angelis G, Coletta M, Paolucci E, Stornaiuolo A, Cossu G, Mavilio F (1998) Muscle regeneration by bone marrow-derived myogenic progenitors. Science 279:15281530

Ikegawa S, Ohashi H, Nishimura G, Kim KC, Sannohe A, Kimizuka M, Fukushima Y, Nagai T, Nakamura Y (1998) Novel and recurrent COMP (cartilage oligomeric matrix protein) mutations in pseudoachondroplasia and multiple epiphyseal dysplasia. Hum Genet 103:633-638

Kitahara O, Furukawa Y, Tanaka T, Kihara C, Ono K, Yanagawa R, Nita ME, Takagi T, Nakamura Y, Tsunoda T (2001) Alterations of gene expression during colorectal carcinogenesis revealed by cDNA microarrays after laser-capture microdissection of tumor tissues and normal epithelia. Cancer Res 61:3544-3549

Komori T, Yagi H, Nomura S, Yamaguchi A, Sasaki K, Deguchi K, Shimizu Y, Lefebvre V, Huang W, Harley VR, Goodfellow PN, de Crombrugghe B (1997) SOX9 is a potent activator of the chondrocyte-specific enhancer of the pro alpha1(II) collagen gene. Mol Cell Biol 17:2336-2346

Luscher B, Larsson LG (1999) The basic region/helix-loop-helix/ leucine zipper domain of Myc proto-oncoproteins: function and regulation. Oncogene 18:2955-2966

Newton G, Weremowicz S, Morton CC, Copeland NG, Gilbert DJ, Jenkins NA, Lawler J (1994) Characterization of human and mouse cartilage oligomeric matrix protein. Genomics 24:435-439

Ochi K, Mori T, Toyama Y, Nakamura Y, Arakawa H (2002) Identification of semaphorin $3 \mathrm{~B}$ as a direct target of $\mathrm{p} 53$. Neoplasia 4:82-87

Ochi K, Chen G, Ushida T, Gojo S, Segawa K, Tai H, Ueno K, Ohkawa H, Mori T, Yamaguchi A, Toyama Y, Hata J, Umezawa A (2003) Use of isolated mature osteoblasts in abundance acts as desired-shaped bone regeneration in combination with a modified poly-DL-lactic-co-glycolic acid (PLGA)collagen sponge. J Cell Physiol 194:45-53

Ono K, Tanaka T, Tsunoda T, Kitahara O, Kihara C, Okamoto A, Ochiai K, Takagi T, Nakamura Y (2000) Identification by cDNA microarray of genes involved in ovarian carcinogenesis. Cancer Res 60:5007-5011

Pittenger MF, Mackay AM, Beck SC, Jaiswal RK, Douglas R, Mosca JD, Moorman MA, Simonetti DW, Craig S, Marshak DR (1999) Multilineage potential of adult human mesenchymal stem cells. Science 284:143-147

Rosen ED, Spiegelman BM (2000) Molecular regulation of adipogenesis. Annu Rev Cell Dev Biol 16:145-171

Ross M, Romrell L, Kaye G (1995) Histology: a text and atlas, 3rd edn. Williams \& Wilkins, Baltimore

Rudnicki JA, Brown AM (1997) Inhibition of chondrogenesis by Wnt gene expression in vivo and in vitro. Dev Biol 185:104-118

Saito-Hisaminato A, Katagiri T, Kakiuchi S, Nakamura T, Tsunoda T, Nakamura Y (2002) Genome-wide profiling of gene expression in 29 normal human tissues with a cDNA microarray. DNA Res 9:35-45

Spain BH, Bowdish KS, Pacal AR, Staub SF, Koo D, Chang CY, Xie W, Colicelli J (1996) Two human cDNAs, including a homolog of Arabidopsis FUS6 (COP11), suppress G-protein- and mitogen-activated protein kinase-mediated signal transduction in yeast and mammalian cells. Mol Cell Biol 16:6698-6706

Stieber A, Mourelatos Z, Chen YJ, Le Douarin N, Gonatas NK (1995) MG160, a membrane protein of the Golgi apparatus which is homologous to a fibroblast growth factor receptor and to a ligand for E-selectin, is found only in the Golgi apparatus and appears early in chicken embryo development. Exp Cell Res 219:562-570

Takeda S, Bonnamy JP, Owen MJ, Ducy P, Karsenty G (2001) Continuous expression of Cbfal in nonhypertrophic chondrocytes uncovers its ability to induce hypertrophic chondrocyte differentiation and partially rescues Cbfa1-deficient mice. Genes Dev 15:467-481

Thur J, Rosenberg K, Nitsche DP, Pihlajamaa T, Ala-Kokko L, Heinegard D, Paulsson M, Maurer P (2001) Mutations in cartilage oligomeric matrix protein causing pseudoachondroplasia and multiple epiphyseal dysplasia affect binding of calcium and collagen I, II, and IX. J Biol Chem 276:6083-6092

Tufan AC, Daumer KM, Tuan RS (2002) Frizzled-7 and limb mesenchymal chondrogenesis: effect of misexpression and involvement of N-cadherin. Dev Dyn 223:241-253

Wade PA (2001) Transcriptional control at regulatory checkpoints by histone deacetylases: molecular connections between cancer and chromatin. Hum Mol Genet 10:693-698 